\title{
Consciência da doença na doença de Alzheimer: resultados preliminares de um estudo longitudinal
}

\author{
Awareness of disease in Alzheimer's disease: preliminary results of a longitudinal study \\ Maria Fernanda Barroso de Sousa ${ }^{1}$, Raquel Luiza Santos ${ }^{1}$, Cynthia Arcoverde², Marcia Dourado ${ }^{3}$, Jerson Laks ${ }^{4}$
}

1 Especialista em Psicogeriatria do Instituto de Psiquiatria da Universidade Federal do Rio de Janeiro (IPUB/UFRJ).

2 Mestranda em Saúde Mental do IPUB/UFRJ.

3 Doutora em Ciências da Saúde do IPUB/UFRJ.

${ }_{4}$ Professor-associado da Faculdade de Ciências Médicas da Universidade do Estado do Rio de Janeiro (UERJ).

Recebido: $29 / 3 / 2010$ - Aceito: $21 / 6 / 2010$

\begin{abstract}
Resumo
Contexto: A presença de déficits cognitivos e dos sintomas psicológicos e do comportamento nas demências torna o fenômeno da consciência da doença um objeto de estudo bastante complexo. Esse fenômeno tem sido mais investigado em estudos de corte transversal do que em estudos de corte longitudinal. Objetivo: Comparar o comprometimento da consciência da doença na doença de Alzheimer (DA) ao longo de seis meses. Método: Ao longo de seis meses, 18 pacientes com DA leve foram avaliados por meio da Escala de Avaliação do Impacto Psicossocial da Demência (AIPD), do Miniexame do Estado Mental (MEEM), do Estadiamento Clínico da Demência (CDR), da Escala Cornell para Depressão na Demência (Cornell), da Escala Qualidade de Vida na Doença de Alzheimer (QdV-DA) - versão paciente e do Questionário de Atividades Funcionais (Pfeffer). Os cuidadores foram avaliados com a Escala Zarit Burden Interview (Zarit) e a QdV-DA - versão cuidador. Resultados: Ao final de seis meses, houve declínio no grau de consciência da doença $(\mathrm{p}=0,02)$, no estado cognitivo ( $\mathrm{p}<0,01)$, nas atividades funcionais $(\mathrm{p}<0,01)$ e no estadiamento clínico da doença $(\mathrm{p}<0,01)$ e aumento dos sintomas depressivos $(\mathrm{p}<0,01)$. Conclusão: Há comprometimento da consciência da doença e déficits cognitivos e funcionais à medida que a gravidade da demência aumenta.
\end{abstract}

Sousa MFB, et al. / Rev Psiq Clín. 2011;38(2):57-60

Palavras-chave: Demência, anosognosia, consciência do déficit, insight.

\begin{abstract}
Background: The presence of cognitive deficits and behavioral and psychological symptoms in dementia makes the phenomenon of awareness of disease a rather complex object of study. This phenomenon has been most studied in cross-sectional studies than in longitudinal studies. Objective: To compare awareness of disease in Alzheimer's patients over six months. Methods: Early-stage Alzheimer's disease patients $(\mathrm{n}=18)$ were evaluated over six months using Scale of Psychosocial Impact of the Diagnosis of Dementia (ASPIDD), Mini Mental State Examination (MMSE), Clinical Dementia Rating (CDR), Cornell Depression Scale in Dementia (Cornell), Quality of Life in Alzheimer's Disease (QoL-AD - patient's version) and Pfeffer Functional Activities Questionnaire (PFAQ) and caregivers were assessed using Zarit Burden Interview (Zarit) and QoL-AD - caregiver's version. Results: At six months' observation, there was a decline in the degree of awareness of disease $(p=0,02)$, in cognitive status $(p<0.01)$, in functional activities $(p<0.01)$, in clinical staging of the dementia ( $<<0.01)$ and an increase in depressive symptoms $(\mathrm{p}<0.01)$. Discussion: As the severity of dementia increases, there is also impaired awareness of disease, together with cognitive and functional deficits.
\end{abstract}

Sousa MFB, et al. / Rev Psiq Clín. 2011;38(2):57-60

Keywords: Dementia, anosognosia, awareness of deficit, insight.

\section{Introdução}

A consciência da doença é definida como a capacidade de perceber em si e/ou nas atividades da vida diária alterações ocasionadas por déficits associados ao processo de adoecimento ${ }^{1}$. No conceito de consciência da doença não é necessário que o reconhecimento do adoecimento se estenda a todos os sintomas ou à avaliação do tipo e da gravidade da doença. A consciência da doença é um fenômeno constituído por três dimensões: (1) a capacidade de reconhecer um déficit específico; (2) a resposta emocional relacionada às dificuldades ou ao déficit e (3) a capacidade de compreender o impacto ou as consequências do comprometimento nas atividades de vida diária ${ }^{2,3}$. Nos processos demenciais, a consciência da doença é flutuante e pode estar comprometida parcial ou totalmente $e^{4,5}$.

O fenômeno da consciência da doença é um objeto de estudo bastante complexo. Essa complexidade se associa às barreiras colocadas pelos déficits cognitivos na avaliação das percepções dos pacientes sobre si e sobre as atividades de vida diária, às deficiências apresentadas pelos métodos de avaliação, além da interação em sua etiologia de fatores que envolvem aspectos neuroanatômicos e aspectos psicossociais ${ }^{6}$.

Grande parte das pesquisas sobre consciência da doença se baliza em estudos transversais. Os achados indicam que há relação entre consciência da doença e disfunção no lobo frontal, depressão e gravidade clínica da demência ${ }^{7}$. Entretanto, uma avaliação em um ponto no tempo pode ser problemática, pois a consciência da doença é um fenômeno flutuante. Isso ocorre uma vez que os fatores biológicos, psicológicos e sociais influenciam a percepção dos déficits, o que acarreta uma variação da percepção do paciente8.

Ao mesmo tempo, os estudos longitudinais são inconclusivos e controversos ${ }^{9-19}$. Alguns estudos longitudinais sugerem associação $0^{9,12-16,19}$ entre a consciência da doença e a gravidade clínica da demência, enquanto em outros estudos não foi evidenciada essa relação ${ }^{10,11,17,18}$. A avaliação longitudinal da consciência da doença na demência apresenta dificuldades relacionadas ao acompanhamento de um grande número de pacientes ${ }^{11}$, métodos de avaliação varia- 
dos $^{11}$, avaliação de vários tipos de demência em um mesmo estudo ${ }^{12} \mathrm{e}$ participantes que apresentam diferença estatística pouco significativa entre as aplicações ${ }^{11,12,17,19}$. Mais estudos longitudinais são necessários para poder oferecer informações mais completas sobre o processo da consciência da doença e sobre a relação entre consciência da doença e outras variáveis como mudanças na pontuação do estado cognitivo por meio do Miniexame do Estado Mental (MEEM) ou aspectos sociodemográficos ${ }^{9,11}$.

Este estudo longitudinal tem como objetivo comparar o comprometimento da consciência ao longo de seis meses em pacientes com doença de Alzheimer (DA) 20.

\section{Método}

\section{Participantes}

Pacientes com DA possível/provável de acordo com os critérios do Manual Diagnóstico e Estatístico de Transtornos Mentais - quarta edição (DSM-IV) e National Institute of Neurological and Communicative Diseases and Stroke/Alzheimer's Disease and Related Disorders Association (NINCDS-ADRDA) $(\mathrm{n}=25)$ foram incluídos por admissão consecutiva em atendimento no ambulatório do Centro para Doença de Alzheimer e Outros Transtornos Mentais na Velhice do Instituto de Psiquiatria da Universidade Federal do Rio de Janeiro (CDA/UFRJ). Os pacientes foram encaminhados após o diagnóstico por um psiquiatra. O diagnóstico clínico foi feito por meio de anamnese com pacientes e familiares, aplicação de testes de rastreio cognitivo, gravidade da demência, avaliação da presença de sintomas psicológicos e do comportamento, exames laboratoriais e de imagem. Todos os pacientes estavam em uso de anticolinesterásicos. Os pacientes apresentaram comorbidades como hipotireoidismo, hipercolesterolemia, hipertensão arterial sistêmica, tabagismo, prolapso da válvula mitral e diabetes. A avaliação longitudinal da consciência da doença foi realizada em dois tempos, sendo a segunda seis meses após a avaliação de base.

Os critérios de inclusão foram: escores do MEEM entre 12 e 26; Demência leve (CDR1) considerada pelo Estadiamento Clínico das Demências (CDR) e escore inferior a 7 na Escala Cornell para Depressão na Demência (Cornell). Foram excluídos os pacientes com doenças psiquiátricas atual ou prévia, afasia, história de alcoolismo e epilepsia.

\section{Instrumentos}

1. Avaliação do estado cognitivo: o MEEM ${ }^{21}$ foi utilizado para avaliar o estado cognitivo. O MEEM é um instrumento de rastreamento cognitivo que avalia memória, orientação, atenção, concentração, linguagem e praxia. A pontuação varia entre 0 e 30 . O pior desempenho é o escore 0 . O intervalo de escores de 12 a 26 utilizado neste estudo se deve à necessidade de adequação dos pontos de corte e escolaridade $^{21}$ e também porque esse intervalo corresponde a quadros moderados a leves de transtorno cognitivo.

2. Avaliação da gravidade clínica da $D A$ : o $C^{22}$ é uma escala de avaliação do estadiamento da demência, que varia de 0 (sem demência) a 5 (demência grave) de acordo com o comprometimento cognitivo, comportamental e nas atividades da vida diária. Foi utilizado o protocolo completo.

3. Avaliação da presença de sintomas depressivos: a avaliação de depressão foi feita por meio da Escala de Depressão na Demência de Cornell ${ }^{23}$, que avalia sinais relacionados ao humor, distúrbios de comportamento, sinais físicos, funções circadianas (sono, alimentação) e pensamentos depressivos. A pontuação acima de 7 denota presença de depressão leve, moderada ou grave.

4. Avaliação da qualidade de vida na doença de Alzheimer: a qualidade de vida na DA foi avaliada pela Escala Qualidade de Vida na Doença de Alzheimer (QdV-DA) ${ }^{24}$, que avalia 13 itens quantificados em quatro pontos. A pontuação 1 é atribuída à qualificação ruim, pontuação 2 a regular, a pontuação 3 a bom e a pontuação 4 a excelente. A pontuação varia entre 13 (ruim) e 52 (excelente). Essa escala possui versões para o paciente e para o cuidador.
5. Avaliação das atividades funcionais: as atividades funcionais foram avaliadas pelo Questionário de Atividades Funcionais (Pfeffer) ${ }^{25,26}$. Essa escala avalia 10 itens em relação à funcionalidade mediante o grau de independência para realização das atividades instrumentais de vida diária. A pontuação varia entre 0 e 30 . A partir do escore 3 indica a presença de prejuízo funcional e quanto maior a pontuação, mais dependente é o paciente.

6. Avaliação de sobrecarga em cuidadores: a avaliação de sobrecarga em cuidadores foi feita pela Escala Zarit Burden Interview (Zarit) ${ }^{27}$. Essa escala é composta por 22 itens e avalia o quanto as atividades do cuidado têm impacto sobre a vida social, o bem-estar físico e emocional e as finanças do cuidador. As respostas de cada item podem variar de 0 a 4 pontos, desde nunca até sempre.

7. Avaliação da consciência da doença: a consciência da doença foi avaliada por meio da Escala de Avaliação do Impacto Psicossocial do Diagnóstico de Demência (AIPD) ${ }^{1,3}$, composta por 35, perguntas divididas em cinco áreas: consciência do déficit, relação social, relação familiar, atividades de vida diária e relação afetiva. Essa escala avalia a discrepância entre o relato do paciente e do cuidador. A consciência da doença foi considerada preservada quando tanto os déficits cognitivos, principalmente a perda de memória, quanto os prejuízos causados pela DA na vida cotidiana eram mencionados espontaneamente pelo paciente e havia concordância entre as histórias do paciente/cuidador. Quando o paciente apenas percebia o déficit cognitivo ou os prejuízos na vida diária e existiam discrepâncias entre a história dele e a do cuidador, a consciência da doença foi considerada parcialmente comprometida. A ausência de consciência da doença foi avaliada quando o paciente não percebia/ negava a presença de déficits cognitivos e os prejuízos causados pela doença e existiam discrepâncias entre paciente e cuidador.

Os pacientes e cuidadores foram entrevistados em conjunto por um psicólogo para a entrevista sociodemográfica e aplicação das escalas de avaliação.

O estudo foi aprovado pelo Comitê de Ética do IPUB/UFRJ e todos os pacientes e familiares assinaram o termo de consentimento livre e esclarecido.

\section{Análise estatística}

As variáveis investigadas (MEEM, Pfeffer, Cornell, QdV-DA e Zarit) não apresentaram distribuição normal e foram analisadas por testes não paramétricos. Com isso, a comparação do momento baseline e o segundo momento da avaliação de um mesmo grupo foi realizada por meio do teste de Wilcoxon e do teste qui-quadrado $\left(\mathrm{X}^{2}\right)$ para comparar as variáveis categóricas AIPD e CDR.

No segundo momento da avaliação, procurou-se comparar dois grupos (grupo 1, que não alterou a consciência da doença, e grupo 2, que alterou a consciência da doença). Para a comparação das variáveis dependentes entre os grupos (grupo $1 \mathrm{x}$ grupo 2), foi realizado o teste de Mann-Whitney-U. A significância estatística foi estabelecida para $\mathrm{p} \leq 0,05$. As análises foram realizadas com o software SPSS versão 15.0.

\section{Resultados}

O grupo de pacientes com DA era, inicialmente, constituído por 25 participantes, todos apresentando demência leve e sem diferenças sociodemográficas. No entanto, no segundo momento de avaliação, sete participantes foram excluídos do estudo: um paciente apresentou sintomas psicóticos; um paciente evoluiu para uma síndrome demencial mista e cinco familiares recusaram-se a retornar à segunda avaliação. Ao final, foram avaliados, longitudinalmente, 18 pacientes.

Dentre os pacientes avaliados, 12 eram mulheres $(66,6 \%)$ A idade dos pacientes foi de 77,7 $\pm 7,18$ (63-88) anos. A escolaridade foi baixa ( 4 anos ou menos) em 7 pacientes $(38,8 \%)$, intermediária ( 7 anos a 11 anos) em 10 pacientes $(55,5 \%)$ e alta (12 anos ou mais) em 1 paciente $(5,5 \%)$. O tempo de duração da doença foi de $5,2 \pm 2,84$ ( 1 ano a 9 anos). A maioria dos cuidadores é de mulheres $(88,8 \%)$. Os cuidadores familiares foram: filhas (50\%), esposas (22,2\%), filho 
(5,5\%), sobrinha (5,5\%) e marido (5,5\%). A escolaridade foi intermediária (8 a 11 anos) em 10 cuidadores $(55,5 \%)$ e alta (12 anos e mais) em 8 cuidadores $(44,4 \%)$.

A variação do grau de consciência da doença foi avaliada em baseline e no segundo momento. No momento baseline, 7 pacientes $(38 \%)$ apresentaram consciência da doença preservada, 10 pacientes (55\%), consciência da doença parcial e 1 paciente (5\%), consciência da doença ausente. No segundo momento, encontraram-se consciência da doença preservada em 1 paciente (5\%), consciência da doença parcial em 13 pacientes (72\%) e consciência da doença ausente em 4 pacientes (22\%).

Todos os pacientes, no momento baseline, tinham DA leve (CDR1). No segundo momento, foram encontrados 11 pacientes $(60 \%)$ que se mantiveram como CDR1 e 7 pacientes $(38 \%)$ que evoluíram para CDR2.

Não foram encontradas diferenças significativas entre consciência da doença, gênero, idade e tempo de doença. O grau de consciência da doença apresentou diferença estatisticamente significativa entre o momento baseline e o segundo momento de avaliação ( $\mathrm{p}=$ $0,02)$. Foram encontradas também diferenças significativas entre o momento baseline e o segundo momento na avaliação do estado cognitivo, nas atividades funcionais, na presença de sintomas depressivos e no estadiamento clínico da doença, como pode ser observado na tabela 1 .

Tabela 1. Diferenças entre as variáveis dependentes no baseline e segundo momento

\begin{tabular}{l|c|c|c}
\hline & $1^{\text {a }}$ avaliação & $2^{\text {a }}$ avaliação & p valor \\
\hline MEEM (escore) & $24,0(16-25)$ & $16,0(14-25)$ & $0,00^{*}$ \\
\hline Pfeffer (escore) & $12,5(5-19)$ & $16,5(7-24)$ & $0,00^{*}$ \\
\hline Cornell (escore) & $2,0(1-3)$ & $4,0(2-7)$ & $0,00^{*}$ \\
\hline OdV-paciente (escore) & $34,0(30-38)$ & $33,0(30-37)$ & $0,60^{*}$ \\
\hline Zarit (escore) & $31,0(19-41)$ & $30,0(21-46)$ & $0,43^{*}$ \\
\hline OdV-cuidador (escore) & $31,0(25-37)$ & $28,0(27-33)$ & $0,20^{*}$ \\
\hline
\end{tabular}

MEEM: Miniexame do Estado Mental; Pfeffer: Questionário de Atividades Funcionais; Cornell: Escala Cornell para Depressão na Demência; OdV-paciente: Escala Qualidade de Vida na Doença de Alzheimer; Zarit: Escala Zarit Burden Interview; OdV-cuidador: Escala Qualidade de Vida na Doença de Alzheimer.

* Valores expressos em mediana e intervalo interquartílico.

Como a amostra utilizada foi heterogênea, procurou-se comparar no segundo momento o estado cognitivo geral, atividades funcionais, qualidade de vida e sobrecarga do cuidador. Os 18 pacientes foram divididos em dois grupos, independentemente do seu estadiamento clínico. O grupo $1(\mathrm{n}=9)$ foi composto por pacientes que não apresentaram alteração da consciência da doença e o grupo $2(n=9)$ foi formado por pacientes que apresentaram alteração da consciência da doença. Foi encontrada diferença estatisticamente significativa no segundo momento da avaliação entre o grupo 1 e o grupo $2 \mathrm{em}$ relação ao estado cognitivo geral $(\mathrm{p}<0,01)$, presença de sintomas depressivos $(\mathrm{p}=0,05)$ e atividades funcionais $(\mathrm{p}=0,05)$ como indicado na tabela 2.

Tabela 2. Comparação entre estado cognitivo, funcional, depressão, qualidade de vida e sobrecarga do cuidador em relação ao grupo 1 e 2 após seis meses

\begin{tabular}{l|c|c|c}
\hline & Grupo 1 $(n=9)$ & Grupo 2 $(n=9)$ & \multirow{2}{*}{ p valor } \\
\cline { 2 - 3 } & Manteve AIPD & Alterou AIPD & $0,01^{*}$ \\
\hline MEEM (escore) & $25(22-26)$ & $15(9-16)$ & $0,05^{*}$ \\
\hline Pfeffer (escore) & $18(11-28)$ & $28(18-30)$ & $0,05^{*}$ \\
\hline Cornell (escore) & $4(1-7)$ & $4(4-7)$ & 0,79 \\
\hline Zarit (escore) & $25(23-47)$ & $38(15-41)$ & 0,90 \\
\hline OdV-paciente (escore) & $32(30-34)$ & $36(28-38)$ & 0,24 \\
\hline OdV-cuidador (escore) & $26(25-30)$ & $22(19-25)$ & \\
\hline
\end{tabular}

MEEM: Miniexame do Estado Mental; Pfeffer: Questionário de Atividades Funcionais; Cornell: Escala Cornell para Depressão na Demência; CDR: Estadiamento Clínico da Demência; Zarit: Escala Zarit Burden Interview; OdV-paciente: Escala Qualidade de Vida na Doença de Alzheimer; OdV-cuidador: Escala Qualidade de Vida na Doença de Alzheimer.

* Diferença significativa no segundo momento da avaliação entre o grupo 1 e 0 grupo 2.

\section{Discussão}

Neste estudo preliminar, demonstrou-se comprometimento da consciência da doença em uma avaliação longitudinal, corroborando resultados encontrados em outros estudos $9,12,13,15,16,19$.

Os pacientes com DA, frequentemente, apresentam comprometimento da consciência da doença em relação a suas dificuldades cognitivas e funcionais ${ }^{9}$. No entanto, a variedade de métodos de avaliação e seleção de sujeitos pode ocasionar resultados diversos, pois se sabe que a consciência da doença não é um constructo unitário e tem variadas manifestações clínicas ${ }^{8,9,11}$. Realizou-se uma avaliação de consciência da doença mais completa, incluindo funcionalidade e qualidade de vida, uma vez que, normalmente, as avaliações feitas na área restringem-se apenas a aspectos relacionados ao comprometimento cognitivo $0^{9,12,15-18}$. Esses achados sugerem que o comprometimento da consciência da doença em pacientes com DA leve estaria relacionado ao agravamento dos estados cognitivo $9,12,15,19$ e funcional ${ }^{10,19}$. Esses achados consubstanciam a hipótese de que o comprometimento da consciência da doença na DA estaria relacionado à gravidade clínica da doença $a^{9,12,15,19}$.

A presença de estados depressivos em pacientes com DA leve tem sido relatada em diversos estudos com resultados conflitantes $19,28,29$. Foram excluídos neste estudo pacientes com depressão, na medida em que esse quadro clínico pode ser um fator de confundimento do déficit cognitivo na demência. Entretanto, houve aumento dos sintomas depressivos entre o momento baseline e o segundo momento de avaliação. Alguns estudos ${ }^{10,12}$ relatam que o aumento dos estados depressivos na demência poderia ser considerado como uma reação emocional ante o reconhecimento de algum aspecto do adoecimento. Por outro lado, a depressão pode aguçar nos indivíduos a noção de que algo está errado ou indo mal, propiciando maior reconhecimento de doença. Esses são aspectos que merecem ser aprofundados em estudos posteriores.

O conceito de qualidade de vida tem se constituído em uma das chaves na assistência a pacientes com $\mathrm{DA}^{28}$. A avaliação da qualidade de vida tem sido utilizada, principalmente, para mensuração do reconhecimento por parte do paciente e de seu cuidador de aspectos considerados subjetivos como saúde, amizades, bem-estar e satisfação ${ }^{28}$. Em nosso estudo, observou-se que a avaliação da qualidade de vida por parte dos cuidadores estaria relacionada a ausência ou diminuição dos sintomas depressivos dos pacientes. Variações no humor do paciente são fatores que interferem na percepção do cuidador em relação à qualidade de vida do paciente ${ }^{28}$. Um dado interessante seria o fato de os cuidadores, apesar da sobrecarga de cuidados, não avaliarem a qualidade de vida dos pacientes como negativa. A avaliação que o cuidador faz do estado geral do paciente normalmente tem sido dependente do seu grau de sobrecarga e presença de sintomas depressivos ${ }^{30}$. Em nosso estudo, não se encontrou variação na sobrecarga do cuidador, a qual pode ainda ser considerada como relativamente baixa. Isso pode estar relacionado ao local onde foi realizado o estudo: um centro de referência para DA, no qual cuidadores recebem continuamente informações e orientações quanto à rotina de cuidados e manejo do paciente e seus déficits. Por outro lado, a ausência de diferenças na avaliação da qualidade de vida por parte do paciente pode estar relacionada ao próprio comprometimento da consciência da doença, na medida em que pacientes com DA, com a progressão da doença, tendem a subestimar a presença de seus déficits cognitivos e funcionais, superestimando seu desempenho quando avaliados em testes objetivos ${ }^{13}$.

A operacionalização do constructo consciência da doença merece discussão, visto que muitas vezes paciente e cuidador não concordam quanto à gravidade dos déficits, em virtude de fatores como presença de sobrecarga do cuidador. A análise da discrepância entre pacientes e cuidadores sugere que os pacientes tendem a subestimar suas dificuldades ou os cuidadores a superestimar os déficits dos pacientes, por causa do seu grau de sobrecarga ${ }^{3}$. Entretanto, os cuidadores ainda são considerados os melhores informantes sobre as alterações cognitivas e do comportamento na demência ${ }^{3,30}$. O método de avaliação mais utilizado na área são os questionários de discrepância entre os relatos dos pacientes e cuidadores $9,10,13,15,16,18,19$. Com o objetivo de controlar 
essas variáveis, avaliou-se a relação entre a sobrecarga do cuidador e a percepção da qualidade de vida do paciente por parte deste.

Algumas limitações deste estudo podem ser apontadas. Os resultados devem ser interpretados com certa cautela, pois a amostra é pequena, fato que esperamos sanar em futuro próximo. Outra limitação se refere à ausência de um grupo controle. Entretanto, até esse momento e até onde é do nosso conhecimento, este é o primeiro estudo brasileiro de avaliação longitudinal da consciência da doença na DA.

Este estudo demonstra que há comprometimento significativo da consciência da doença ao longo de seis meses de observação de pacientes com DA. Um dado relevante está relacionado à realização de uma avaliação mais acurada do estado cognitivo e funcional. Esses dados podem ser úteis para auxiliar a comunicação entre pacientes e familiares, entre profissionais de saúde e pacientes e cuidadores, para o planejamento de atividades de reabilitação e orientação familiar e ainda para a determinação de capacidades civis e legais dos pacientes com demência.

\section{Referências}

1. Dourado MCN, Marinho V, Soares C, Engelhardt E, Laks J. Awareness of disease in dementia: development of a multidimensional rating scale. Dement Neuropsychol. 2007;1:74-80.

2. Flashman L. Disorders of awareness in neuropsychiatric syndromes: an update. Curr Psychiatry Rep. 2002;4:346-53.

3. Dourado MCN, Laks J, Rocha M, Soares C, Leibing A, Engelhardt E. Consciência da doença na demência: resultados preliminares em pacientes com doença de Alzheimer leve e moderada. Arq Neuropsiquiatr. 2005;63:114-8.

4. Howorth P, Saper J. The dimensions of insight in people with dementia. Aging Ment Health. 2003;7(2):113-22.

5. Zannetti O, Vallotti B, Frisoni G, Geroldi C, Bianchetti A, Pasqualetti P, et al. Insight in dementia: when does it occur? Evidence for a nonlinear relationship between insight and cognitive status. J Gerontol Psychol Sci. 1999;54B(2):100-6.

6. Clare L. Managing threats to self: awareness in early stage Alzheimer's disease. Social Sci Med. 2003;57:1017-29.

7. Dourado MCN, Laks J, Leibing A, Engelhardt E. Consciência da doença na demência. Rev Psiq Clín. 2006;33(6):313-21.

8. Lamar M, Lasarev MR, Libon DJ. Determining levels of unawareness in dementia research. J Neuropsychiatry Clin Neurosci. 2002;14(4):430-7.

9. Akai T, Haruo H, Hirofumi S, Tomohiko S, Toshihiko I. Longitudinal patterns of unawareness of memory deficits in mild Alzheimer's disease. Geriatr Gerontol Int. 2009;9:16-20.

10. Montañés $P$, Quintero E. La Anosognosia en la enfermedad de Alzheimer: una aproximación clínica para el estudio de la conciencia. Rev Latinoam Psicol. 2007;39(1):63-73.

11. Clare L, Wilson BA. Longitudinal assessment of awareness in early-stage Alzheimer's disease using comparable questionnaire-based and performance-based measures: a prospective one-year follow-up study. Aging Ment Health. 2006;10(2):156-65.

12. Aalten P, Van Valen E, De Vugt ME, Lousberg R, Jolles J, Verhey FRJ. Awareness and behavioral problems in dementia patients: a prospective study. Int Psychogeriatr. 2006;18(1):3-17.

13. Vogel A, Hasselbalch SG, Andersen BB, Mortensen EL, Waldemar G. Patient versus informant reported quality of life in the earliest phases of Alzheimer's disease. Int J Geriatric Psychiatry. 2006;21:1132-8.
14. Marshall GA, Kaufer DI, Lopez OL, Rao GR, Hamilton RL, Dekosky ST. Right prosubiculum amyloid plaque density correlates with anosognosia in Alzheimer's disease. J Neurol Neurosurg Psychiatry. 2004;75:1396-400.

15. Vogel A, Hasselbalch SG, Gade A, Ziebell M, Waldemar G. Cognitive and functional neuroimaging correlates for anosognosia in Mild Cognitive Impairment and Alzheimer's disease. Int J Geriatric Psychiatry. 2005;20(3):238-46.

16. Vogel A, Hasselbalch SG, Ziebell M, Gade A, Stokholm J, Waldemar G. Awareness of deficits in mild cognitive impairment and Alzheimer's disease: do MCI patients have impaired insight? Dement Geriatr Cogn Disord. 2004;17(3):181-7.

17. Arkin S, Mahendra, N. Insight in Alzheimer's patients: results of a longitudinal study using three assessment methods. Am J Alzheimers Dis Other Demen. 2001;16:211-24.

18. Sevush S. Relationship between denial of memory deficit and dementia severity in Alzheimer disease. Neuropsychiatry Neuropsychol Behav Neurol. 1999;12(2):88-94.

19. Derouesné C, Thibault S, Lagha-Pierucci S, Baudouin-Madec V, Ancri D, Lacomblez L. Decreased awareness of cognitive deficits in patients with mild dementia of the Alzheimer type. Int J Geriatric Psychiatry. 1999;14:1019-30.

20. Gallucci JN, Tamelini MG, Forlenza OV. Diagnóstico diferencial das demências. Rev Psiq Clín. 2005;32(3):119-30.

21. Laks J, Batista EMR, Guilherme ERL, Contino AL, Faria ME, Figueira I, et al. O mini-exame do estado mental em idosos de uma comunidade: dados parciais de Santo Antonio de Pádua, Rio de Janeiro. Arq Neuropsiquiatr. 2003;61(3B):782-5.

22. Maia ALG, Godinho C, Ferreira ED, Almeida V, Schuh A, Kaye J, et al. Aplicação da versão brasileira da Escala de Avaliação Clínica da Demência (Clinical Dementia Rating - CDR) em amostras de pacientes com demência. Arq Neuropsiquiatr. 2006;64(2-B):485-9.

23. Carthery-Goulart MT, Areza-Fegyveres R, Schultz RR, Okamoto I, Caramelli P, Bertolucci PHF, et al. Versão brasileira da Escala Cornell de Depressão em Demência (Cornell Depression Scale in Dementia). Arq Neuropsiquiatr. 2007;65(3b):916-9.

24. Novelli MMPC. Validação da escala de qualidade de vida (QdV-DA) para pacientes com doença de Alzheimer e seus respectivos cuidadores familiares [tese]. São Paulo (SP): Faculdade de Medicina/USP; 2006.

25. Pfeffer RI, Kurosaki TT, Harrah Jr CH, Chance JM, Filos S. Measurement of functional activities in older adults in the community. J Gerontol. 1982;37:323-9.

26. Nitrini R, Caramelli P, Bottino CMC, Damasceno BP, Brucki SMD, Anghinah R. Diagnóstico de doença de Alzheimer no Brasil: avaliação cognitiva e funcional. Recomendações do Departamento Científico de Neurologia Cognitiva e do Envelhecimento da Academia Brasileira de Neurologia. Arq NeuroPsiquiatr. 2005;63(3a):720-7.

27. Scazufca M. Versão brasileira da escala Burden Interview para avaliação de sobrecarga em cuidadores de indivíduos com doenças mentais. Rev Bras Psiquiatr. 2002;24(1):12-7.

28. Logsdon RG, Gibbons LE, Mc Curry SM, Teri L. Assessing quality of life in older adults with cognitive impairment. Psychosom Med. 2002;64:510-9.

29. Smith CA, Henderson VW, Mc Cleary CA, Murdock GA, Buckwalter JG. Anosognosia and Alzheimer's disease: the role of depressive symptoms in mediating impaired insight. J Clin Exp Neuropsychol. 2000;22(4):437-44.

30. Karlawish JH, Casarett D, Klocinski J, Clark CM. The relationship between caregivers' global ratings of Alzheimer's disease patients' quality of life, disease severity, and the caregiving experience. J Am Geriatr Soc. 2001;49:1066-70. 